\title{
СЕМАНТИЧЕСКИЙ АНАЛИЗ НОМЕНКЛАТУРЫ ЗАПАСНЫХ СОСТАВНЫХ ЧАСТЕЙ СЛОЖНОЙ ТЕХНИЧЕСКОЙ СИСТЕМЫ
}

\section{SEMANTIC ANALYSIS \\ OF THE NOMENCLATURE \\ OF SPARE PARTS OF A COMPLEX TECHNICAL SYSTEM}

\section{Golikov \\ E. Mikhailova}

Summary. The article deals with the current problem of lack of spare parts and components of complex technical systems to ensure their technical inspection and operation during the entire warranty period.

The analysis is carried out in order to identify products that cannot be considered as an object of inclusion in the range of spare parts. These products include power products that have high strength reserves in their design, which cause their high reliability, as well as products that can only be replaced in the factory.

Keywords: mechanical engineering, component, spare parts, methodology, kit, complex, reliability, maintenance, complex technical systems.

Голиков Владислав Андреевич

Ассистент, Московский авиационный институт (национальный исследовательский университет) fordik08@mail.ru

Михайлова Екатерина Вячеславна Ассистент, Московский авиационный институт (национальный исследовательский университет) evmihailova@inbox.ru

Аннотация. Рассмотрена актуальная на сегодняшний день проблема недостатка запасных составных частей и узлов сложных технических систем для обеспечения их технического осмотра и эксплуатации в течении всего срока гарантийного использования.

Анализ проводится с целью выделения изделий, которые не могут рассматриваться как объект включения в номенклатуру запасных частей. К таким изделиям относятся силовые изделия, в конструкции которых заложены высокие запасы прочности, обуславливающие их высокую надежность, а также изделия, замена которых возможна лишь в заводских условиях.

Ключевые слова: машиностроение, составная часть, запасные части, методика, комплект, комплекс, надежность, техническое обслуживание, сложные технические системы.

\section{Введение}

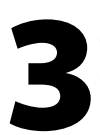

апасные составные части (3С4) сложной технической системы предназначены для замены отказавших или выработавших ресурс изделий, а так же для:

- обеспечения использования одной системы в течение расчетного срока эксплуатации с требуемым критерием надежности;

- технического обеспечения системы в период расчетного срока эксплуатации;

- контроля технического состояния системы.

Состав ЗСЧ разрабатывается с учетом надежности составных частей (СЧ) подлежащих замене и имеющих техническую возможность их замены, а также остальных ограничений по массе, габаритам и т.д.

\section{Метолика опрелеления номенк^атуры запасных частей}

Интенсивность отказов изделий при работе и хранении берется из справочников по характеристикам надежности изделий [1].

В таблице 1 приведена номенклатура исходных данных для расчета состава 3СЧ.

Принятая допустимая вероятность а отсутствия запасной части в комплекте представляет собой веро- 
Таблица 1. Исходные данные

\begin{tabular}{|c|c|}
\hline Наименование & Обозначение \\
\hline Расчетный срок эксплуатации 3СЧ & $M_{3 c 4}$ \\
\hline Гарантийная наработка, цикл работы & B \\
\hline Допустимая вероятность отсутствия запасной части в ЗСЧ & $\mathrm{T}$ \\
\hline
\end{tabular}

Таблица 2. Исходные переменные

\begin{tabular}{|l|l|}
\hline Наименование и размерность & Обозначение \\
\hline 1 Интенсивность отказов изделий ј-ой позиции номенклатуры при работе, $1 / 4$ & $\delta$ \\
\hline 2 Интенсивность отказов изделий ј-ой позиции номенклатуры при хранении, 1/4 & $\delta$ \\
\hline 3 Суммарная наработка изделий ј-ой позиции номен- клатуры за расчетный срок эксплуатации 3СЧ, ч & $\mathrm{M}_{p j}$ \\
\hline 4 Суммарное время хранения изделий ј-ой позиции номенклатуры за расчетный срок эксплуатации 3СЧ, ч & $\mathrm{M}_{p j}$ \\
\hline 5 Ожидаемое среднее число отказов изделий ј-ой позиции номенклатуры & $\mathrm{m}_{j}$ \\
\hline 6 Количество изделий ј-ой позиции номенклатуры, установленные в системе, шт. & $\mathrm{n}_{j}$ \\
\hline 7 Количество запасных частей изделий ј-ой позиции номенклатуры, шт. & $\mathrm{X}_{j}$ \\
\hline 8 Остаток, определяющий необходимость срочного пополнения, шт. & $\mathrm{X}_{c n}$ \\
\hline
\end{tabular}

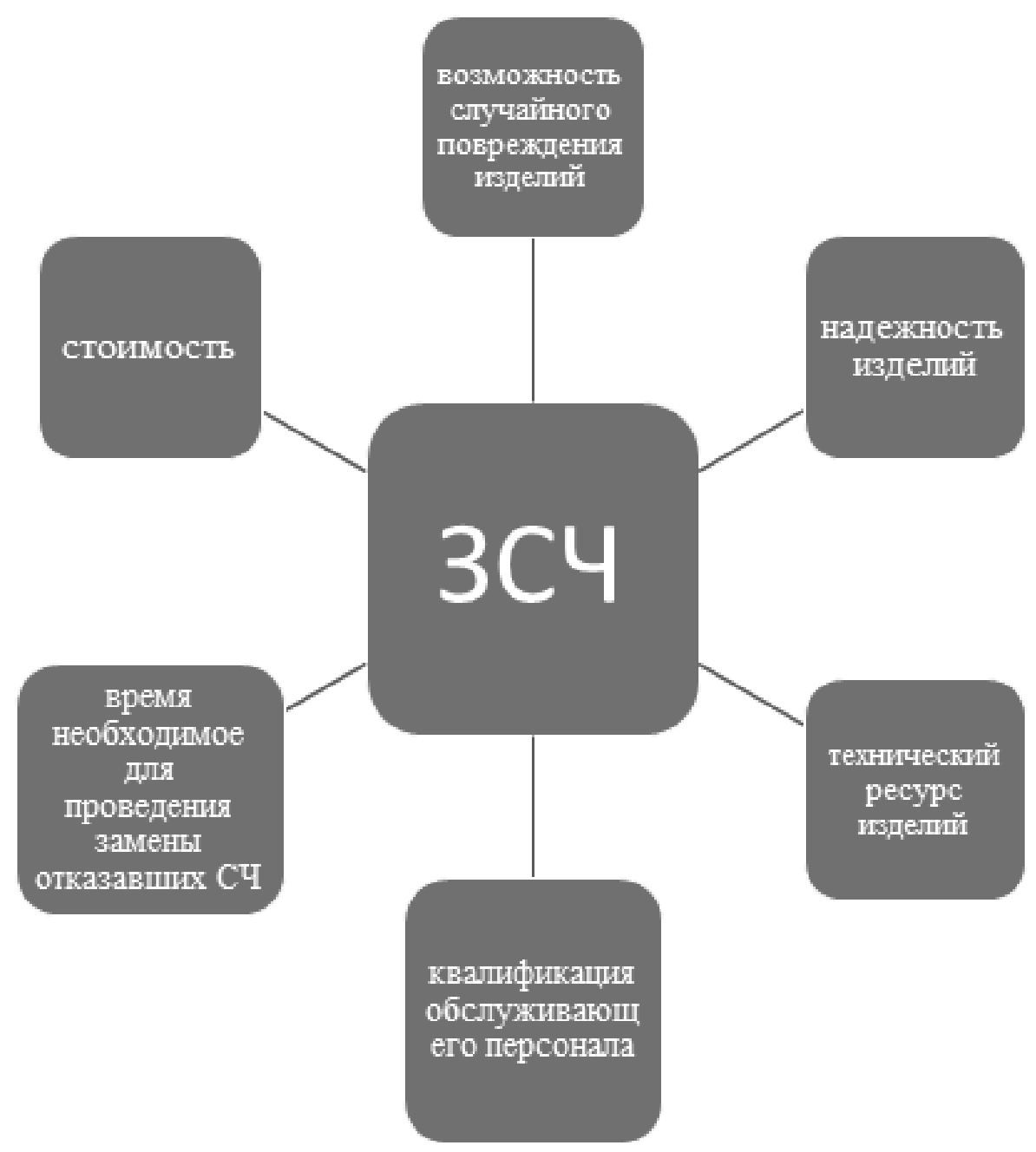

Рис. 1 
Таблица 3. Остаток срочного пополнения

\begin{tabular}{|l|l|l|l|l|l|}
\hline$X_{j}$ & 1 & 2 & 3 & 4 & 5 \\
\hline$X_{c n}$ & 0 & 0 & 0 & 0 & 1 \\
\hline
\end{tabular}

ятность того, что за расчетный срок эксплуатации ЗСЧ количество отказавших изделий превысит количество аналогичных изделий, находящихся в 3Сч [2-3].

В таблице 2 приведены исходные переменные необходимые для расчета состава ЗСЧ.

Номенклатура 3СЧ определяется методом инженерного анализа с учетом [4-6] следующих факторов (рис. 1):

В номенклатуру включаются изделия [7-9]:

- непосредственно влияющие на работоспособность всей системы и не являющиеся практически безотказными;

- отказ которых ведет к возникновению аварийной ситуации и назначенный ресурс которых меньше назначенного ресурса системы;

- необходимые для проведения ремонтно-восстановительных работ, технического обслуживания и контроля технического состояния;

- которые в результате эксплуатации могут быть повреждены.

\section{Метолика определения количества запасных частей}

Количество запасных частей $X_{j}$ для каждой позиции определяется исходя из величины вероятности отсутствия запасной части в ЗСЧ, $\alpha$ и ожидаемого среднего числа отказов изделий $m_{j}$.

Ожидаемое среднее число отказов $j$-ой позиции номенклатуры определяется по формуле:

$m_{j}^{2}=\left(\delta_{p j} \cdot M_{p j}+\delta_{x p j} \cdot M_{x p j}\right)^{2}$

где $m_{j}, \delta_{p j}, M_{p j}, \delta_{x p j}, M_{x p j}-$ см. таблицу 2.

Суммарное время хранения $M_{x p j}$ изделий $j$-ой позиции номенклатуры определяется по формуле:

$M_{x p j}=M_{3 C \psi} \cdot n_{j}-M_{p j}$

где $M_{3 С 4}-$ см. таблицу 1 ; $n_{j}-$ см. таблицу 2.

\section{Метолика определения минИМа^ьногО остатКа срочного пополнения}

Под остатком, определяющим необходимость срочного пополнения, понимается количество ЗСЧ каждой позиции номенклатуры, по достижении которого необходимо пополнение комплекта [10-11].

Остаток, определяющий необходимость срочного пополнения, назначается с целью постоянного обеспечения в процессе эксплуатации запасными частями на случай, когда их фактический расход превышает расчетный [12].

Остаток, определяющий необходимость срочного пополнения Хсп устанавливается:

- при количестве запасных частей по каждой позиции номенклатуры $\mathrm{Xj} \leq 5$ в соответствии с таблицей 3.

- при количестве запасных частей по каждой позиции номенклатуры по следующей формуле [13],

$X_{c n}=0,2 X_{j}$

где $X_{c n}, X_{j}-$ см. таблица 2.

\section{Зак^ючение}

При верном определении номенклатуры запасных составных частей, их количества и необходимого минимального остатка срочного пополнения может быть получена рекомендация по составу комплекта удовлетворяющего заданным ограничениям по массе, габаритам, надежности и возможности технической замены, а так же минимально необходимого для:

- обеспечения использования одной системы в течение расчетного срока эксплуатации с требуемым критерием надежности;

- технического обеспечения в период расчетного срока эксплуатации;

к контроля технического состояния

\section{ЛИТЕРАТУРА}

1. Ю.К. Беляев, В. А. Богатырев, В. В. Болотин и др. Надежность технических систем: Справочник Под ред. И. А. Ушакова.

2. ГОСТ 27.310-95 Надежность в технике. Анализ видов, последствий и критичности отказов. Основные положения.—Введ. 1995-10-12. - М.: Издательство стандартов, 1996. 
3. ГОСТ Р ИСО 9000-2015 Системы менеджмента качества. Основные положения и словарь

4. ГОСТ Р ЕН 9100-2011 Системы менеджмента качества организации авиационной, космической и оборонных отраслей промышленности.

5. ОСТ 134-1028-2012 Требования к системам менеджмента качества предприятий, участвующих в создании, производстве и эксплуатации изделий.

6. Амосов, А. Г. Проблематика маневренности автопоездов при проектировании / А. Г. Амосов, М. Ю. Куприков, В. А. Голиков // Известия Тульского государственного университета. Технические науки. — 2018. — № 9.—С. 342-349.

7. Бойцов Б. В. Жизненный цикл и реализация летательного аппарата //В.Д. Борисов, Н. М. Киселев, В. Г. Подколзин //М.: Изд-Во МАИ — 2005. — С. 520.

8. Cheng $S$. and Cebon D. Improving roll stability of articulated heavy vehicle using active semi-trailer steering. - Vehicle System Dynamics: International Journal of Vehicle Mechanics and Mobility, Vol 46, Supplement: 373-388, 2008.

9. Михалев В.В. Инновационные подходы к вопросам расчета комплекта современных средств. Сборник «Инновационная деятельность в вооруженных силах российской федерации. Труды всеармейской научно-практической конференции -2015. - С. 308.

10. Гришин, А. С. Разработка методики прогнозирования потребности предприятий автосервиса в запасных частях: диссертация канд. Техн. Наук/МАДИ.M., 2005.-C. 197.

11. Кузнецов, Е.С. Техническая эксплуатация автомобилей: учеб. Для вузов. 4-е издание, переработанное и дополненное / Е. С. Кузнецов [и др.].— М.: Наука, 2001.

12. ГОСТ 27.507-2017. Надежность военной техники. Оценка и расчет запасов в комплектах ЗИП.- Введ. 2015-12-10.- М.: СТАНДАРТИНФОРМ, 2017.

13. Михейчик И. В. Сравнительный анализ методов и методик для обоснования комплектов ЗИП. Сборник: Автомобиле- и тракторостроение. Материалы Международной научно-практической конференции в 2-ух томах. - 2018 -С. 214.

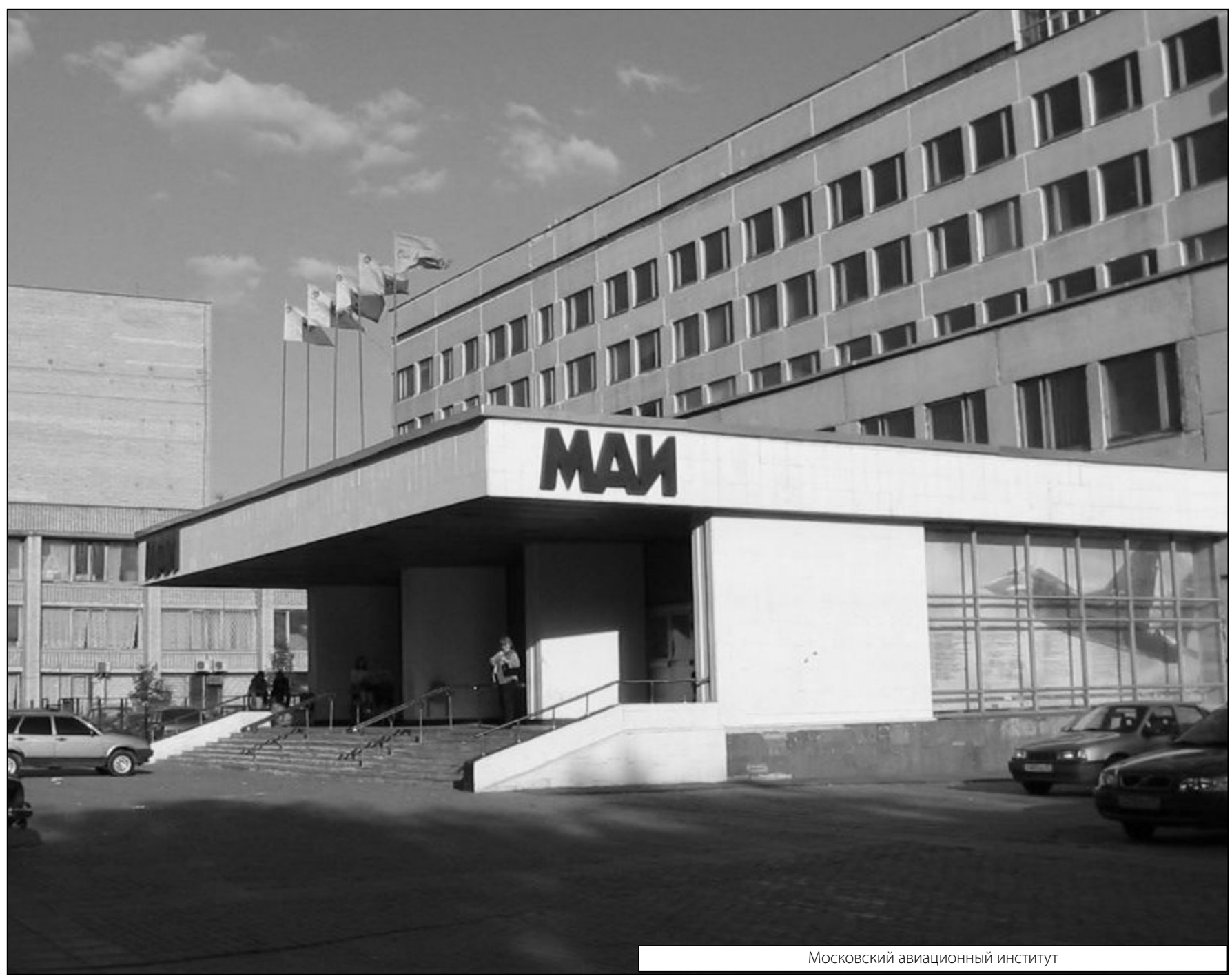

